\title{
UNIFORM DISTRIBUTION IN LOCALLY COMPACT ABELIAN GROUPS $\left({ }^{1}\right)$
}

\author{
BY \\ I. D. BERG, M. RAJAGOPALAN AND L. A. RUBEL( ${ }^{2}$ )
}

Introduction. The notion of a uniformly distributed sequence on a compact group was introduced and studied by Eckmann in [1], and has since been the object of much research. Recently, this notion was extended to locally compact groups by Rubel in [9]. Our principal interest here is in the following two questions. First, when does a locally compact Abelian group $G$ possess a uniformly distributed sequence? Second, when is there an associated compact group $G^{\sim}$ and a continuous homomorphism $\varphi: G \rightarrow G^{\sim}$, such that $\varphi(G)$ is dense in $G^{\sim}$, with the property that $\left\{g_{v}\right\}$ is uniformly distributed in $G$ if and only if $\left\{\varphi\left(g_{v}\right)\right\}$ is uniformly distributed in $G^{\sim}$ ? We call such a group $G^{\sim}$ a $D$-compactification of $G$. We obtain solutions to these and related problems.

In this introduction, we present various definitions and preliminary results and describe briefly the main results of the paper. In $\$ 1$, we prove some structural lemmas. In $\S 2$, we consider the existence of uniformly distributed sequences on $G$. Finally, in $\S 3$, we consider the existence of $D$-compactifications of $G$.

By $G$, we will denote a locally compact Abelian group. We will, in general, assume that all groups are locally compact Abelian (abbreviated LCA) groups. We do not require that our groups be first-countable. Indeed, even when we start with first-countable groups $G$, we will be forced to consider certain "compactifications" which will rarely be first-countable. Throughout this paper, we shall assume the continuum hypothesis, denoting by $c$ the cardinal number of the continuum. If $A$ is a set, we denote the cardinal number of $A$ by card $A$.

Characters of $G$ are supposed to be continuous characters unless otherwise stated. We will denote the trivial character by 1 . The dual group of $G$ will be denoted by $\Gamma$ or by $G^{\wedge}$.

If $G$ is an LCA group, we will call a compact group $H$ a compactification of $G$ if there is a continuous homomorphism from $G$ onto a dense subgroup of $H$. For example, $\bar{G}$, the Bohr compactification of $G$, (defined as the dual of $\Gamma_{d}$, where

Received by the editors January 24, 1967 and, in revised form, May 2, 1967.

(1) A preliminary report on this paper was presented as an address by the third author to the Österreichischen Mathematischen Gesellschaft on March 25, 1966.

$\left.{ }^{2}\right)$ The research of the first two authors was partially sponsored by the National Science Foundation under grant NSF GP-5370. The research of the third author was sponsored in part by the Air Force Office of Scientific Research under grant number AFOSR 460-63, and also in part by a Senior Postdoctoral Fellowship from the National Science Foundation. 
$\Gamma_{d}$ is $\Gamma$ in the discrete topology) is a compactification of $G$. The trivial group $\{0\}$ is a compactification of any LCA group $G$.

In order to give a canonical construction for all compactifications of an LCA group $G$, and to avoid isomorphism problems, we construct each compactification $H$ as the compact dual of a subgroup of $\Gamma_{d}$. Hence when a character of $H$ occurs we freely identify it with a character of $G$.

When an LCA group $H$ is a quotient group of $G$, or if $H$ contains such a quotient group, in such a way that there is a distinguished homomorphism from $G$ into $H$, we will use the notation $\varphi_{H}$ for this homomorphism, or simply $\varphi$ if there is no possibility of confusion. Sometimes, by a slight abuse of notation, if $H$ is a closed subgroup of $G$, we write $\varphi_{H}$ rather than $\varphi_{G / H}$.

A subgroup $H$ of $G$ is said to be of compact index if $G / H$ is compact. A function on $G$ is said to be periodic if it is constant on the cosets of some subgroup of compact index. It is easy to see that a character $\chi$ is periodic if and only if $G /(\operatorname{ker} \chi)$ is compact. We denote the collection of periodic characters by $\Gamma^{p}$. It is not always the case (see $\S 1$ ) that $\Gamma^{p}$ is a subgroup of $\Gamma$. By $\bar{G}^{p}$ we denote the compact dual of the subgroup of $\Gamma_{d}$ generated by $\Gamma^{p}$, and call $\bar{G}^{p}$ the periodic compactification of $G$. It is easy to see that $\bar{G}^{p}$ is actually a compactification of $G$ and that $\bar{G}^{p}$ is always a quotient of $\bar{G}$.

For example, if $G=R$ is the real numbers in the usual topology, we see that $\bar{G}^{p}=\bar{G}$ since every character of $R$ is periodic. If, on the other hand, $G=R_{d}$, the reals in the discrete topology, then $\bar{G}^{p}=\{0\}$, since 1 is the only periodic character. However in this case, $\bar{G}$ is a decidedly nontrivial compact group, being the dual of the discrete group consisting of all characters of $R$, whether continuous or not in the usual topology. If $G=Z$, the integers in the discrete topology, then the periodic characters form a group. In this case $\bar{G}^{p}$ is smaller than the Bohr compactification -namely $\bar{G}^{p}$ is the so-called universal monothetic Cantor group. That is, $\bar{G}^{p}$ is the compact dual of the discrete group of rational numbers modulo 1.

Definition. Let $G$ be a group, $f$ a complex-valued function on $G$, and $\left\{g_{v}\right\}$ a sequence of elements of $G$. By $\left\langle f,\left\{g_{v}\right\}\right\rangle$ we denote the following limit, if it exists:

$$
\left\langle f,\left\{g_{v}\right\}\right\rangle=\lim _{n \rightarrow \infty} \frac{1}{n} \sum_{v=1}^{n} f\left(g_{v}\right)
$$

Uniform distribution in compact groups has been the object of a great deal of study. The interested reader is referred to Volume 16 of Compositio Mathematica which is entirely devoted to uniform distribution, or our references [1] and [3], for access to the literature. We choose as our definition of uniform distribution on a compact group a characterization which is due to Eckmann. A standard alternative definition can be found on p. 253 of [9] where it is referred to as a proposition of Eckmann's.

Definition (EckmanN). Let $G$ be a compact Abelian group. We say that the 
sequence $\left\{g_{v}\right\}$ of elements of $G$ is uniformly distributed in $G$ if for each continuous complex-valued function $f$ on $G,\left\langle f,\left\{g_{v}\right\}\right\rangle=\int f d \mu$, where $\mu$ is Haar measure on $G$.

If we write $\left\langle f,\left\{g_{v}\right\}\right\rangle=a$, it is understood that $\left\langle f,\left\{g_{v}\right\}\right\rangle$ exists. We use the abbreviation u.d. for uniformly distributed.

With this definition the following basic theorem, which can be found in [1], is immediate.

THEOREM (WEYL CRITERION). If $G$ is a compact Abelian group, then $\left\{g_{v}\right\}$ is uniformly distributed in $G$ if and only if, for each nontrivial character $\chi,\left\langle\chi,\left\{g_{v}\right\}\right\rangle=0$.

Definition (RUBEL [9]). Let $G$ be an LCA group. We say that the sequence $\left\{g_{v}\right\}$ of elements of $G$ is uniformly distributed in $G$ if for each subgroup $H$ of compact index in $G,\left\{\varphi_{H}\left(g_{v}\right)\right\}$ is uniformly distributed in $G / H$.

The Weyl criterion now clearly assumes the following form:

THEOREM (WEYL CRITERION). If $G$ is an LCA group then $\left\{g_{v}\right\}$ is uniformly distributed in $G$ if and only if for each nontrivial periodic character $\chi,\left\langle\chi,\left\{g_{v}\right\}\right\rangle=0$.

If $G=R$, then $\left\{g_{v}\right\}$ is u.d. if and only if $\left\{\varphi\left(g_{v}\right)\right\}$ is u.d. in $\bar{R}$, the Bohr compactification of $R$. This is the case if and only if $\left\{\operatorname{tg}_{v}\right\}$ is u.d. $\bmod 1$ for each $t \in R, t \neq 0$. If $G=R_{d}$ then, trivially, any sequence $\left\{g_{v}\right\}$ is u.d. If $G=Z$, then $\left\{g_{v}\right\}$ is u.d. if and only if $\left\{\varphi\left(g_{v}\right)\right\}$ is u.d. in $\bar{G}^{p}$. However, $\left\{g_{v}\right\}$ may be u.d. in $Z$ even though $\left\{\psi\left(g_{v}\right)\right\}$ is not u.d. in $\bar{G}$, even though both $\varphi$ and $\psi$ are one-to-one. It is easy to see (in accordance with a definition of Niven [6]) that $\left\{g_{v}\right\}$ is u.d. in $Z$ if and only if the $g_{v}$ fall into each arithmetic progression with limiting frequency equal to the arithmetic density of the progression.

We will often use, sometimes without special reference, the following two well-known results. The first is the structure theorem for LCA groups [10, Theorem 2.4.1] and the second is a special case of a theorem of Kakutani [5].

StruCture Theorem. Every LCA group $G$ has an open subgroup $G_{1}$ of the form $G_{1}=H \times R^{n}$ where $H$ is compact and $R^{n}$ is Euclidean n-space in the usual topology; $G_{1}$ is also closed. Furthermore, $G / G_{1}$ is discrete.

TheOrem A (KATUtANi). A compact Abelian group $G$ is separable (that is, there exists $a$ dense sequence in $G$ ) if and only if card $\Gamma \leqq c$.

Definition. An LCA group $G$ is $K$-separable if there exists a sequence $\left\{g_{v}\right\}$ in $G$ such that, for every subgroup $H$ of compact index in $G$, the sequence $\left\{\varphi_{H}\left(g_{v}\right)\right\}$ is dense in $G / H$.

We may now describe briefly the main results of this paper.

A necessary and sufficient condition that the periodic characters of $G$ form a group is that either every discrete quotient of $G$ be of bounded order (which happens precisely when every character of $G$ is periodic) or $G$ be totally disconnected.

Next, $G$ admits a u.d. sequence if and only if $G$ is $K$-separable. This is not an 
entirely satisfactory result. The following result, although not quite complete, is perhaps more satisfactory: If card $\Gamma^{p} \leqq c$, then $G$ admits a u.d. sequence. If $\Gamma^{p}$ is a group and $G$ admits a u.d. sequence, then card $\Gamma^{p} \leqq c$. For any cardinal $n$, there exists a group $G$ such that card $\Gamma^{p} \geqq n$ yet such that $G$ admits a u.d. sequence.

Finally, there is the question of the existence and uniqueness of a $D$-compactification of $G$. Let us make the mild assumption that $G$ admits a u.d. sequence, since otherwise the subject of $D$-compactifications becomes trivial. Under this assumption, $G$ has a $D$-compactification if and only if $\Gamma^{p}$ is a group, and if $\Gamma^{p}$ is a group then there is a unique $D$-compactification, namely $\bar{G}^{p}$. In this case, the suitability of $\bar{G}^{p}$ is clear; the proof of uniqueness requires some work.

We remark that Rajagopalan and Rotman [7], have characterized those discrete groups that admit a u.d. sequence of the form $\{n g\}, n=1,2,3, \ldots$, and that Rajagopalan [8] has recently extended this result to arbitrary LCA groups.

\section{The structure of $\Gamma^{p}$.}

LEMMA 1.1. Let $G$ be an LCA group and let $G_{0}$ denote the identity component of $G$. Let $\chi$ be a character of $G$ and let $H$ denote the kernel of $\chi$. If $\chi$ does not annihilate $G_{0}$, then $G / H=T$, the circle group in the usual topology, and $\chi$ is periodic. If $\chi$ annihilates $G_{0}$ then $G / H$ is discrete, and in this case $\chi$ is periodic if and only if the range of $\chi$ is finite.

Proof. We first note that $\chi$ induces a one-to-one continuous homomorphism $\chi^{\sim}$ from $G / H$ into $T$; indeed if $g^{\sim}$ is the image in $G / H$ of $g$ in $G$ then we define $\chi^{\sim}\left(g^{\sim}\right)$ $=\chi(g)$.

Suppose that $G_{0} \nsubseteq H$. Then $G / H$ has a nontrivial connected component of the identity, $G_{1}$. If $G / H$ is connected then the range of $\chi^{\sim}$ is $T$ since the image of $G / H$ must be connected. Because $G / H$ is connected it is easily seen to be $\sigma$-compact and therefore $\chi^{\sim}$ must be open [4, Theorem 5.29], and hence $G / H$ is (homeomorphically) isomorphic to $T$. In general, if $G / H$ is not totally disconnected then $G_{1}$ must map isomorphically onto a connected subset of $T$ and hence $G / H=G_{1}=T$ as above. Therefore $\chi$ is periodic.

Now suppose that $G_{0} \subseteq H$. Then $G / H$ is totally disconnected; moreover since every neighborhood of 0 in $G / H$ contains an open subgroup and since there is a neighborhood of 0 in $T$ that contains no nontrivial subgroup it follows that $G / H$ is discrete. Hence $G / H$ is compact and $\chi$ is periodic if and only if the range of $\chi$ is finite.

This completes the proof of Lemma 1.1.

LEMMA 1.2. Let $G$ be an LCA group. Then all the characters of $G$ are periodic if and only if every discrete quotient of $G$ is of bounded order.

Proof. We have seen that if there is a nonperiodic character $\chi$ in $\Gamma$, then $G / H$ is discrete and infinite. Since $G / H$ is algebraically a subgroup of $T$, there must be 
elements of $G / H$ of arbitrarily high (possibly infinite) order. On the other hand, if $G$ has a discrete quotient $K$ that is not of bounded order, then there is a character $\chi$ of $K$ whose range is infinite. This is not difficult to see-it follows by a simple category argument. But we may consider, in the obvious way, $\chi$ as a character on $G$, and we see that $G /(\operatorname{ker} \chi)$ is therefore discrete and infinite, since $G /(\operatorname{ker} \chi)$ is the same as $K /(\operatorname{ker} \chi)$, so that $\chi$ is not periodic. This completes the proof of Lemma 1.2.

THEOREM 1. Let $G$ be an LCA group. Then the periodic characters of $G$ form a group if and only if either every discrete quotient of $G$ is of bounded order, or else $G$ is totally disconnected.

Proof. If $G$ is totally disconnected, then by Lemma $1.1, \Gamma^{p}$ is the set of characters whose range is finite, and these clearly form a group. If every discrete quotient of $G$ is of bounded order, then by Lemma 1.2, every character is periodic so that the periodic characters again form a group.

Suppose now that $G$ has a nontrivial connected component of the identity, say $G_{0}$, and a nonperiodic character $\chi_{1}$. Then by Lemma 1.1, $\chi_{1}$ must annihilate $G_{0}$. Now, since the characters on $G$ separate the elements of $G$, we may choose a character $\chi_{2}$ which does not annihilate $G_{0}$ and therefore must be periodic. Then $\chi_{1} \chi_{2}$ does not annihilate $G_{0}$ and hence $\chi_{1} \chi_{2}$ is periodic. But then $\left(\chi_{1} \chi_{2}\right)\left(\chi_{2}\right)^{-1}$ is a nonperiodic product of periodic characters, and our proof of Theorem 1 is done.

2. The existence of uniformly distributed sequences. We begin with a computational lemma, which is somewhat similar to some results for compact groups; for example, Hlawka's Theorem 7 of [3].

LEMMA 2.1. Let $G$ be an LCA group and let $\Phi$ be a subset of $\Gamma=G^{\wedge}$. Let $H$ be a countable subgroup of $G$ that separates the nontrivial characters in $\Phi$ from 1 . That is, for each nontrivial character $\chi$ in $\Phi$ there is an element $h$ in $H$ such that $\chi(h) \neq 1$. Then there is a sequence $\left\{k_{v}\right\}$ of elements of $H$ such that for each nontrivial character $x$ in $\Phi$, we have $\left\langle\chi,\left\{k_{v}\right\}\right\rangle=0$.

Proof. Let $H=\left\{h_{v}\right\}, \nu=1,2, \ldots$, and consider the following sequence $\left\{k_{v}\right\}$ :

$$
\begin{aligned}
& h_{1}, h_{1} h_{2}, h_{1}^{2} h_{2}, h_{1} h_{2}^{2}, h_{1}^{2} h_{2}^{2}, h_{1} h_{2}^{3}, h_{1}^{2} h_{2}^{3}, h_{1} h_{2}^{4}, h_{1}^{2} h_{2}^{4}, h_{1} h_{2} h_{3}, h_{1}^{2} h_{2} h_{3}, \\
& h_{1}^{3} h_{2} h_{3}, h_{1} h_{2}^{2} h_{3}, h_{1}^{2} h_{2}^{2} h_{3}, h_{1}^{3} h_{2}^{2} h_{3}, h_{1} h_{2}^{3} h_{3}, h_{1}^{2} h_{2}^{3} h_{3}, h_{1}^{3} h_{2}^{3} h_{3}, h_{1} h_{2}^{4} h_{3}, \\
& h_{1}^{2} h_{2}^{4} h_{3}, h_{1}^{3} h_{2}^{4} h_{3}, \ldots, h_{1}^{3} h_{2}^{9} h_{3}, \ldots, h_{1}^{3} h_{2}^{9} h_{3}^{27}, h_{1} h_{2} h_{3} h_{4}, \ldots, \\
& h_{1}^{n} h_{2}^{n 2} \ldots h_{n}^{n n}, \ldots
\end{aligned}
$$

To describe this construction, consider the block that begins with $h_{1} h_{2} \cdots h_{n}$ and ends with $h_{1}^{n} h_{2}^{n^{2}} \cdots h_{n}^{n n}$. The exponent of $h_{1}$ runs in order through the numbers from 1 to $n$ and then cycles. The exponent of $h_{2}$ runs through the numbers from 1 to $n^{2}$ in blocks of $n$ and then cycles. In general, for $j=1, \ldots, n$, the exponent of $h_{j}$ runs through the numbers from 1 to $n^{j}$ in blocks of $n^{k_{j}}$, with $k_{j}=j(j-1) / 2$, and then cycles. 
We give a formula for finding the $p$ th term of this block where $p$ runs from 0 to $n^{n(n-1) / 2}-1$. We write

$$
\begin{array}{ll}
p=p_{1} n+\left(q_{1}-1\right) & \text { where } 1 \leqq q_{1} \leqq n, \\
p_{1}=p_{2} n^{2}+\left(q_{2}-1\right) & \text { where } 1 \leqq q_{2} \leqq n^{2}, \\
\vdots & \\
p_{n-1}=p_{n} n^{n}+\left(q_{n}-1\right) & \text { where } 1 \leqq q_{n} \leqq n^{n} .
\end{array}
$$

Then the $p$ th term of this block is $h_{1}^{q} h_{2}^{q} \cdots h_{n}^{q}$. We then begin the next block with $h_{1} h_{2} \cdots h_{n+1}$.

We will show that this sequence has the required properties. Let $\chi$ be a fixed nontrivial character in $\Phi$, and let $h_{\nu}$ be the first element of $H$ on which $\chi \neq 1$. After a finite number of terms, we can group the above sequence in successive blocks, in each term of which all the factors with indices exceeding $\nu$ are fixed. In each such block, the exponents of $h_{v}$ run from 1 to $p^{v}$ for appropriate $p$. Let us observe that for $p>\nu$, there are several such blocks corresponding to $p$. We will designate such a block by $B_{p}$. Note that $p$ is nondecreasing. Each exponent is repeated $p \cdot p^{2} \cdot \cdots \cdot p^{\nu-1}$ times in each $B_{p}$ for a total block length of $p^{\lambda}$ terms, where $\lambda=$ $v(\nu+1) / 2$. Over this block $B_{p}$, we have

$$
\frac{1}{p^{\lambda}} \sum_{j \in B_{p}} \chi\left(k_{j}\right)=\frac{1}{p^{\lambda}} \sum_{r=1}^{p^{\nu}}\left(p \cdot p^{2} \cdots \cdot \cdot p^{\nu-1}\right) \chi\left(h_{v}^{r}\right) \chi(m)
$$

for some fixed $m$. Hence

$$
\lim _{p \rightarrow \infty} \frac{1}{p^{\lambda}} \sum_{j \in B_{p}} \chi\left(k_{j}\right)=0 .
$$

Since the length of $B_{p}$ divided by the number of terms preceding $B_{p}$ approaches 0 as the block lengths grow larger, and since $|\chi|=1$, we see that $\left\langle\chi,\left\{k_{j}\right\}\right\rangle=0$.

This completes the proof of Lemma 2.1. We will not use the next lemma until the proof of Theorem 4 of $\S 3$.

Lemma 2.2. Let $G$ be an LCA group, let $\Phi$ be a subgroup of $\Gamma=G^{\wedge}$, and let $\chi \in \Gamma \mid \Phi$. That is, $\chi \in \Gamma$ but $\chi \notin \Phi$. Suppose there is a sequence $\left\{x_{\nu}\right\}$ in $G$ such that for each nontrivial $\gamma \in \Phi$ we have $\left\langle\gamma,\left\{x_{v}\right\}\right\rangle=0$. Then if $\Psi$ is the subgroup of $\Gamma$ generated (algebraically) by $\Phi$ and $\chi$, there is a sequence $\left\{y_{v}\right\}$ in $G$ such that for each nontrivial $\gamma \in \Psi$, we have $\left\langle\gamma,\left\{y_{v}\right\}\right\rangle=0$.

Proof. First, we note that for each nontrivial $\gamma \in \Phi$ there is an $x_{v}$ such that $\gamma\left(x_{v}\right) \neq 1$. Since $\Phi$ is a group, for each $\gamma_{1}$ and $\gamma_{2}$ in $\Phi$ there is an $x_{v}$ such that $\gamma_{1}\left(x_{v}\right)$ $\neq \gamma_{2}\left(x_{v}\right)$. Hence for all $\gamma \in \Phi$ except at most one, there is an $x_{v}$ such that $(\gamma \chi)\left(x_{v}\right) \neq 1$. By adjoining one more element $w_{1}$, say, to $\left\{x_{v}\right\}$ we obtain a set separating each character of the form $\gamma \chi$ from 1 . That is, for each $\gamma \in \Phi$, either there is some $x_{v}$ so that $(\gamma \chi)\left(x_{v}\right) \neq 1$ or else $(\gamma \chi)\left(w_{1}\right) \neq 1$. Proceeding similarly by adjoining elements $w_{n}$, we separate all characters of the form $\gamma \chi^{n}$ from 1 , where $n=0, \pm 1, \pm 2, \ldots$ By 
Lemma 2.1, we may arrange a subset of the group generated by $\left\{x_{v}\right\}$ and $\left\{w_{n}\right\}$ into a sequence $\left\{y_{v}\right\}$ such that for each nontrivial $\gamma$ in $\Psi$, we have $\left\langle\gamma,\left\{y_{v}\right\}\right\rangle=0$, and Lemma 2.2 is proved.

TheOREM 2. The LCA group $G$ admits a uniformly distributed sequence if and only if $G$ is $K$-separable.

Proof. Suppose first that $G$ admits the u.d. sequence $\left\{g_{v}\right\}$. Then $\left\{g_{v}\right\}$ is $K$-dense in $G$, in the sense that $\left\{\varphi_{H}\left(g_{v}\right)\right\}$ is dense in $G / H$ for every subgroup $H$ of compact index, since $\left\{\varphi_{H}\left(g_{v}\right)\right\}$ is u.d. in $G / H$ and we recall that in a compact group a u.d. sequence must be dense. Next, suppose that $G$ admits a $K$-dense sequence $\left\{g_{v}\right\}$. Now apply Lemma 2.1 with the choices $\Phi=\Gamma^{p}$ and $H$ as the subgroup of $G$ generated by $\left\{g_{v}\right\}$. The sequence $\left\{k_{v}\right\}$ whose existence is asserted by that lemma is u.d. in $G$ by the Weyl criterion. This completes the proof of Theorem 2 .

The next result is perhaps more interesting. We recall that Theorem 1 gives necessary and sufficient conditions that $\Gamma^{p}$ be a group.

THEOREM 3. Let $G$ be a locally compact Abelian group. If card $\Gamma^{p} \leqq c$ then $G$ admits a uniformly distributed sequence. If $\Gamma^{p}$ is a group and $G$ admits a uniformly distributed sequence, then card $\Gamma^{p} \leqq c$. There exists, for any cardinal number $n$, an LCA group $G$ that admits a uniformly distributed sequence, but such that card $\Gamma^{p} \geqq n$.

Before we begin the proof of Theorem 3 some remarks may be appropriate. We will first show that if card $\Gamma^{p} \leqq c$, then $G$ admits a u.d. sequence. Our proof is based on the structure theorem. At first inspection, the following approach seems promising. From the hypotheses, and Theorem A, we know that there is a dense sequence in $\bar{G}^{p}$. If we could choose this dense sequence as the image of a sequence in $G$, we could then apply Lemma 2.1 and the Weyl criterion to get a sequence that is u.d. in $G$. Unfortunately this promising line does not seem to work, since it is possible for a dense subgroup of a separable group to be nonseparable.

Expanding a little on this theme, suppose we let $G=\prod Z_{2}$ be the product of continuum many copies of the two-element group $Z_{2}$ in the product topology, and let $H=\sum Z_{2}$ be the sum in the discrete topology. Now $H$ plays two roles for us. First, we consider $H$ as a group in its own right, embedded densely in $G$ in the obvious manner; that is, $G$ is a compactification of $H$. Second, we regard $H$ as the dual of $G$ and write $H=G^{\wedge}$. Now $G$ has only $c$ characters, yet there is no sequence of elements in $H$ that separates from 1 all the nontrivial characters of $G$. In particular, there is no sequence in $H$ that is u.d. in $G$. So we see that our result depends on the particular set of characters chosen, namely the periodic ones. Our actual proof uses the next lemma.

Lemma 2.3. Let $G$ be discrete, and suppose that card $\Gamma^{p} \leqq c$. Then $G$ admits a uniformly distributed sequence.

Leaving the proof until later, we give the proof of Theorem 3. 
Proof of Theorem 3. We first show that if card $\Gamma^{p} \leqq c$ then $G$ admits a u.d. sequence. By the structure theorem, $G$ contains a closed open subgroup $H=K \times R^{n}$, where $K$ is compact and $G / H=M$ is discrete. We first note that card $\left(M^{\wedge}\right)^{p} \leqq c$ since a periodic character on $M$ has finite range, and hence considered as a character on $G$ is periodic. By Lemma 2.3 and the Weyl criterion, there is therefore a sequence $\left\{m_{v}\right\}$ in $M$ that separates from 1 the nontrivial periodic characters of $M$. We choose $\left\{x_{v}\right\}$ in $G$ so that $\varphi\left(x_{v}\right)=m_{v}$ : then $\left\{x_{v}\right\}$ separates from 1 those nontrivial periodic characters of $G$ that annihilate $H$.

Now consider $Q$, the discrete group of characters of $H$ generated by the images in $H^{\wedge}$ of members of $\Gamma^{p}$, and note that card $Q \leqq c$. Hence $Q^{\wedge}$, the compact dual of $Q$, is separable by Theorem A. Each character on $H$ has the form $\chi_{1} \cdot \chi_{2}$, where $\chi_{1} \in K^{\wedge}$ and $\chi_{2} \in\left(R^{n}\right)^{\wedge}=R^{n}$; it follows that $Q^{\wedge}$ is a quotient group of $K \times B^{n}$, where $B^{n}$ is the Bohr compactification of $R^{n}$. We now note that the rational points in $R^{n}$ are dense in $B^{n}$. Hence we may choose the separating sequence in $Q^{\wedge}$ to be the images of elements of the form $k \times r$, where $k \in K$ and $r \in R^{n}$. Thus there is a sequence $\left\{h_{v}\right\}$ in $H$ which separates from 1 all the nontrivial periodic characters on $G$ that do not annihilate $H$. Hence we have a countable set in $G$ that separates $\Gamma^{p}$ from 1 , and so by Lemma 2.1 and the Weyl criterion, we can construct from it a u.d. sequence in $G$.

Suppose now that $\Gamma^{p}$ is a group and that $G$ admits a u.d. sequence. Then this sequence maps into a u.d. sequence in $\bar{G}^{p}$ since $\bar{G}^{p}$ has the same periodic characters as $G$. In particular, since $\bar{G}^{p}$ is compact, the image of the sequence is a dense sequence in $\bar{G}^{p}$, and by Theorem $A$, we see that card $\left(\bar{G}^{p}\right)^{\wedge} \leqq c$. Since $\left(\bar{G}^{p}\right)^{\wedge}=\Gamma^{p}$, this proves the second assertion.

For the required example, we choose the group $H$ as a product of so many copies of $R_{d}$, the reals in the discrete topology, that card $H^{\wedge}$ is as great as we please and so that $H$ has no nontrivial periodic characters. Let $T$ be the circle group. Then $G=T \times H$ has as periodic characters those characters of the form $e^{i n \theta} \times \chi$, where $n \in Z, n \neq 0$, and $\chi$ is any character of $H$. By Lemma 1.1 , these characters are periodic because they do not annihilate $T$, the connected component of the identity of $G$. It is easy to see that no other characters are periodic.

Any character of the form $1 \times \chi$ is not periodic, so the periodic characters do not form a group since $1 \times \chi=\left(e^{i \theta} \times \chi\right)\left(e^{-i \theta} \times 1\right)$. Now choose $\left\{y_{v}\right\}$ a sequence that is u.d. in $T$, and let $x=y \times 0$. Then $\left\{x_{v}\right\}$ is u.d. in $G$ since all nontrivial periodic characters of $G$ restrict to nontrivial characters of $T$. Thus $G$ has as many periodic characters as required and yet admits a u.d. sequence. This completes the proof of Theorem 3.

We now must prove Lemma 2.3 which was used in the proof of Theorem 3 . We remark that the converse of Lemma 2.3 also holds, since the periodic characters of a discrete group form a group, and by Theorem 3 the cardinality of this group of characters does not exceed $c$.

Proof of Lemma 2.3. First, $\chi \in \Gamma^{p}$ if and only if $G /(\operatorname{ker} \chi)$ is finite. If $G /(\operatorname{ker} \chi)$ 
has $n$ elements, then $\chi$ may be considered as a character of $G / n G$. Now if card $G / n G$ $\geqq c$ then there are at least $2^{c}$ characters on $G / n G([5])$, each of which, by Lemma 1.2, represents a distinct periodic character on $G$. Since this is impossible, we have that card $(G / n G) \leqq \aleph_{0}$. Now let $\left(g_{n, 1}, g_{n, 2}, \ldots\right)$ be elements in $G$ that map into $\left(\varphi\left(g_{n, 1}\right)\right.$, $\left.\varphi\left(g_{n, 2}\right), \ldots,\right)$, the distinct elements of $G / n G$, and let $H$ be the group generated by $\left\{g_{n, r}: r=1,2, \ldots ; n=1,2, \ldots\right\}$. Then $\varphi(H)$ separates from 1 the nontrivial characters in $\bar{G}^{p}$, and hence by Lemma 2.1 and the Weyl criterion, we may construct a sequence $\left\{h_{v}\right\}$ from $H$ which is u.d. in $G$. This completes the proof of Lemma 2.3.

S. Hartman has introduced in [2] the following notion of uniform distribution in an LCA group $G$; we call it Hartman uniform distribution and use the abbreviation H.u.d.

Definition. The sequence $\left\{g_{v}\right\}$ of elements of $G$ is uniformly distributed in the sense of Hartman if $\left\langle\gamma,\left\{g_{v}\right\}\right\rangle=0$ for each nontrivial $\gamma \in \Gamma$.

It is immediate that a sequence is H.u.d. in $G$ if and only if its image in $\bar{G}$, the Bohr compactification of $G$, is u.d. there. There are certain disadvantages to this notion of uniform distribution. In case $G=R$, the notions of H.u.d. and u.d. are easily seen to coincide, but in case $G=Z$, they are different. In view of Niven's paper [6], the notion of u.d. seems more natural. We observe in passing that Lemma 1.2 gives necessary and sufficient conditions on a group $G$ that the notions of u.d. and H.u.d. coincide, namely that every discrete quotient of $G$ be of bounded order. In particular, by Lemma 1.1, they coincide for all connected groups.

Hartman proves (Theorem 3 of [2]) the following result. (We recall that the weight of a topological space is defined to be the smallest cardinal number $W$ such that the space has a neighborhood basis of cardinality $W$.)

THEOREM (HARTMAN). If $G$ is an LCA connected group with weight not exceeding $c$, then $G$ admits a Hartman uniformly distributed sequence.

The following result generalizes Hartman's theorem. To see that it implies his theorem, observe that a connected LCA group can be written as $K \times R^{n}$, where $K$ is compact. Since by Theorem 2 of [5], the weight of a compact group is the same as the cardinality of its dual, we see that for such a group $G$, the weight of $G$ does not exceed $c$ if and only if the cardinality of $G^{\wedge}$ does not exceed $c$.

Proposition 2.4. Let $G$ be an LCA group. A necessary and sufficient condition that $G$ admit a Hartman uniformly distributed sequence is that card $\Gamma \leqq c$.

Proof. Necessity is immediate since such a sequence must be dense in $\bar{G}$ because $\bar{G}$ is compact. Hence by Theorem A, card $(\bar{G})^{\wedge} \leqq c$ and since $(\bar{G})^{\wedge}=G^{\wedge}$ as a group, we are done. In the other direction, we show first, extending part of Theorem $A$, that if card $\Gamma \leqq c$ then $G$ is separable. This is surely known, but we give the proof for completeness. Once we know that $G$ is separable, we are done, since we can use Lemma 2.1 with $\Phi=G^{\wedge}$ and $H$ the subgroup generated by the dense sequence in $G$ to produce an H.u.d. sequence. Now suppose that card $\Gamma \leqq c$ and apply the 
structure theorem to $G$, writing $G / M=Q$. Any discrete quotient $Q$ of $G$ must have cardinality not exceeding $\aleph_{0}$. For otherwise, let $Q^{\wedge}$ be its compact dual. By a theorem of Kakutani [5, Theorem 1], we would have card $Q^{\wedge}>c$. But $Q^{\wedge}$ is a subgroup of $\Gamma$, which leads to a contradiction. Also, any compact open subgroup $K$ of $G$ must have no more than $c$ characters since $K^{\wedge}$ is isomorphic to a quotient group of $\Gamma$. Thus any such $K$ must be separable, by Theorem $\mathrm{A}$, and of course $R^{n}$ is separable. It follows that $G$ is separable since it is the union of the countably many cosets of $M=K \times R^{n}$, and each of these cosets is open and separable. This completes the proof of Proposition 2.4.

3. The existence and uniqueness of $D$-compactifications. We recall that a $D$ compactification $G^{\sim}$ of the LCA group $G$ is a compactification such that a sequence is u.d. in $G$ if and only if its image is u.d. in $G^{\sim}$.

If $G$ admits no u.d. sequence then $G$ trivially admits many $D$-compactifications. Indeed, if $G$ admits no u.d. sequence then, by Theorem 3 , card $\Gamma^{p}>c$. If we then let $\Lambda$ be any subgroup of $\Gamma$ such that card $\Lambda>c$ and if we let $H$ be the compact dual of $\Lambda$ then $H$ will serve vacuously as a $D$-compactification of $G$ since, by Theorem 3, $H$ admits no u.d. sequence.

If $G$ admits a u.d. sequence, we show in Theorem 4 that $G$ has a $D$-compactification if and only if $\Gamma^{p}$ is a group, and that if $\Gamma^{p}$ is a group then there is a unique $D$-compactification, namely $\bar{G}^{p}$.

We wish to thank R. Doss who simplified the authors' original proof of the following computational lemma.

LEMMA 3.1. Let $G$ be a compact Abelian group such that card $\Gamma \leqq c$ and suppose given a character $\chi_{1}$ in $\Gamma$. Then there exists a sequence $\left\{x_{v}\right\}$ in $G$ such that for each character $\chi$ other than $\chi_{1}, \chi_{1}^{-}$, and 1 , we have $\left\langle\chi,\left\{x_{v}\right\}\right\rangle=0$ but $\left\langle\chi_{1},\left\{x_{v}\right\}\right\rangle \neq 0$.

Proof. First, we construct a sequence $\left\{y_{v}\right\}$ in $G$ such that for each nontrivial character $\chi$ we have $\left\langle\chi,\left\{y_{v}\right\}\right\rangle=0$. This is possible by Theorem 3 and the Weyl criterion. Now let $\Psi=1+\left(\chi_{1}+\chi_{i}^{-}\right) / 2$. We will construct the desired sequence $\left\{x_{v}\right\}$ by rearranging and repeating terms of the sequence $\left\{y_{v}\right\}$ so that for each continuous complex-valued function $f$ on $G$ we have $\left\langle f,\left\{x_{v}\right\}\right\rangle=\int f d m$ where $d m=\Psi d \mu$ and $\mu$ is Haar measure on $G$. This will prove our lemma, since if $\chi \in \Gamma$, then $\int_{G} \chi d m=0$ unless $\chi$ is one of $\chi_{1}, \chi_{1}^{-}$, or 1 , whereas if $\chi$ is one of these three characters then $\int_{G} \chi d m \neq 0$.

We construct our sequence $\left\{x_{v}\right\}$ by blocks, with the $n$th block having $T_{n}$ terms. For fixed $n$, let $p_{v n}=\left[n \Psi\left(y_{v}\right)\right]$, where [t] denotes the integral part of $t$. Then

$$
\left|\Psi\left(y_{v}\right) / n-p_{v n} / n^{2}\right|<1 / n^{2}
$$

and consequently

$$
\sum_{v=1}^{n}\left|\frac{\Psi\left(y_{v}\right)}{n}-\frac{p_{v n}}{n^{2}}\right|<\frac{1}{n}
$$


The $n$th block of our sequence $\left\{x_{v}\right\}$ will be $y_{1}, \ldots, y_{1}, y_{2}, \ldots, y_{2}, \ldots, y_{n}, \ldots, y_{n}$, where $y_{j}$ occurs $p_{j n}$ times as $j$ runs through $1,2, \ldots, n$. This block has $T_{n}$ terms, where

$$
T_{n}=\sum_{v=1}^{n} p_{v n}
$$

Let us label the terms of this block as $z_{1}, z_{2}, \ldots, z_{N}$, where $N=T_{n}$. Since $\left\langle\Psi,\left\{y_{v}\right\}\right\rangle$ $=1$, we see that $\lim n^{-2} T_{n}=1$. Also we see that for continuous functions $f$,

$$
\begin{aligned}
\mid \frac{1}{n^{2}} \sum_{j=1}^{T_{n}} f\left(z_{j}\right) & -\frac{1}{n} \sum_{v=1}^{n} \Psi\left(y_{v}\right) f\left(y_{v}\right) \mid \\
= & \left|\frac{1}{n^{2}} \sum_{v=1}^{n} p_{v n} f\left(y_{v}\right)-\frac{1}{n} \sum_{v=1}^{n} \Psi\left(y_{v}\right) f\left(y_{v}\right)\right| \leqq \frac{1}{n} \sup \left|f\left(y_{v}\right)\right|,
\end{aligned}
$$

and it follows directly from the uniform distribution of $\left\{y_{v}\right\}$ that

$$
\lim _{n \rightarrow \infty} \frac{1}{T_{n}} \sum_{j=1}^{T_{n}} f\left(z_{j}\right)=\int_{G} f(x) \Psi(x) d \mu(x) .
$$

But the averages that terminate in the middle of a block behave the same way, since

$$
\lim _{n \rightarrow \infty} \frac{T_{n+1}}{\sum_{v=1}^{n} T_{v}}=0
$$

This completes the proof of Lemma 3.1.

THEOREM 4. Let $G$ be an LCA group which admits a uniformly distributed sequence. Suppose $\Gamma^{p}$ is a group. Then a sequence $\left\{x_{v}\right\}$ of elements of $G$ is uniformly distributed if and only if $\left\{\varphi\left(x_{v}\right)\right\}$, the image of $\left\{x_{v}\right\}$ under the natural homomorphism from $G$ into $\bar{G}^{p}$, is uniformly distributed in $\bar{G}^{p}$. Moreover, $\bar{G}^{p}$ is the only compactification of $G$ with this property. That is, $\bar{G}^{p}$ is the unique D-compactification of $G$. If $\Gamma^{p}$ is not a group, then there is no D-compactification.

Proof. Assume first that $\Gamma^{p}$ is a group. By Theorem 3 , card $\Gamma^{p} \leqq c$. Then since $G$ has as periodic characters precisely the characters of $\bar{G}^{p}$, we see by the Weyl criterion that $\left\{x_{v}\right\}$ is u.d. in $G$ if and only if $\left\{\varphi\left(x_{v}\right)\right\}$ is u.d. in $\bar{G}^{p}$. Suppose now that $H$ is another compactification of $G$, so that $H^{\wedge}$ is a subgroup of $\Gamma$. If $\chi_{1} \in H^{\wedge} \mid \Gamma^{p}$, then by Lemma 2.2 and the Weyl criterion we can find a sequence $\left\{g_{v}\right\}$ in $G$ so that, if $Q$ is the compact dual of the discrete group generated by $\Gamma^{p}$ and $\chi_{1}$, then $\left\{\varphi_{Q}\left(g_{v}\right)\right\}$ is u.d. in $Q$. Then by Lemma 3.1 we may rearrange (with repetitions) $\left\{g_{v}\right\}$ in a new sequence $\left\{k_{v}\right\}$ such that $\left\langle\chi,\left\{k_{v}\right\}\right\rangle=0$ for all $\chi$ in $Q^{\wedge}$ other than $\chi_{1}, \chi_{1}^{-}$, and 1 , but such that $\left\langle\chi_{1},\left\{k_{v}\right\}\right\rangle \neq 0$. So we see that $\left\{k_{v}\right\}$ is u.d. in $G$ but $\left\{\varphi\left(k_{v}\right)\right\}$ is not u.d. in $H$. If, on the other hand, there is a character $\chi_{2} \in \Gamma^{p} \backslash H$, then we apply Lemma 3.1 to get a sequence $\left\{g_{v}\right\}$ such that $\left\langle\chi,\left\{g_{v}\right\}\right\rangle=0$ for all $\chi$ in $\Gamma^{p}$ other than $\chi_{2}, \chi_{2}^{-}$, and 1 , 
and such that $\left\langle\chi_{2},\left\{g_{v}\right\}\right\rangle \neq 0$. Then $\left\{\varphi\left(g_{v}\right)\right\}$ is u.d. in $H$ but not in $G$. Hence, if $H$ is a $D$-compactification then $H=\Gamma^{p}$.

If $\Gamma^{p}$ is not a group, then for any compactification $H$, either $H^{\wedge}$ has a character not in $\Gamma^{p}$ or else $H^{\wedge}$ lacks a character of $\Gamma^{p}$. If $H$ were a $D$-compactification, then $H$ would have to admit a u.d. sequence that was the image of a u.d. sequence in $G$. But then applying Lemma 3.1 and Lemma 2.2, we would obtain either a u.d. sequence in $G$ whose image is not u.d. in $H$ or else a non u.d. sequence in $G$ whose image is u.d. in $H$. Hence $H$ cannot be a $D$-compactification. This completes the proof of Theorem 4.

\section{REFERENCES}

1. B. Eckmann, Uber monothetische Gruppen, Comment. Math. Helv. 16 (1943-1944), 249-263.

2. S. Hartman, Remarks on equidistribution on noncompact groups, Compositio Math. 16 (1965), 66-71.

3. E. Hlawka, Zur formalen Theorie der Gleichverteilung in kompakten Gruppen, Rend. Circ. Mat. Palermo Ser. II 4 (1955), 33-47.

4. E. Hewitt and K. Ross, Abstract harmonic analysis, Springer-Verlag, Berlin, 1963.

5. S. Kakutani, On cardinal numbers related with a compact Abelian group, Proc. Imp. Acad. Tokyo 19 (1943), 366-372.

6. I. Niven, Uniform distribution of sequences of integers, Trans. Amer. Math. Soc. 98 (1961), 52-62.

7. M. Rajagopalan and J. J. Rotman, Monogenic groups, Compositio Math. 18 (1967), 155-161.

8. M. Rajagopalan, Structure of monogenic groups, Illinois J. Math. (to appear).

9. L. A. Rubel, Uniform distribution in locally compact groups, Comment. Math. Helv. 39 (1965), 253-258.

10. W. Rudin, Fourier analysis on groups, Interscience, New York, 1962.

\section{UNIVERSITY OF ILLINOIS,}

URBANA, ILLINOIS 